Horizons philosophiques

\title{
L'épistémologie de Helmholtz et la question du réalisme scientifique
}

\section{Jean Leroux}

Volume 2, numéro 2, printemps 1992

Philosophie et sciences : du concept au réel

URI : https://id.erudit.org/iderudit/800897ar

DOI : https://doi.org/10.7202/800897ar

Aller au sommaire du numéro

Éditeur(s)

Collège Édouard-Montpetit

ISSN

1181-9227 (imprimé)

1920-2954 (numérique)

Découvrir la revue

Citer cet article

Leroux, J. (1992). L'épistémologie de Helmholtz et la question du réalisme scientifique. Horizons philosophiques, 2(2), 83-107.

https://doi.org/10.7202/800897ar d'utilisation que vous pouvez consulter en ligne.

https://apropos.erudit.org/fr/usagers/politique-dutilisation/ 


\section{L'épistémologie de Helmholtz et la question du réalisme scientifique}

Que l'on songe maintenant à ce que deviendrait notre représentation du monde sensible sans cette symbolique de nos sens, si nous étions capables de percevoir directement ce dont le physicien ne s'approche que par une longue chaîne d'inférences. Partout et toujours la même action monotone de forces moléculaires attractives et répulsives, pas d'autre diversité que le changement tout sec des relations numériques, pas de lumière, pas de couleur, pas de son, pas de chaleur.

Hermann von Helmholtz'

Les travaux de Helmholtz en géométrie physique, la sémiotique reliée à sa théorie de la perception et, plus généralement, linfluence kantienne de son épistémologie n'ont pas manqué d'attirer l'attention des philosophes. Cependant, son attitude à l'égard de la thèse réaliste en science n'a à peu près pas été commentée ${ }^{2}$ et c'est ce

1. (1852) “Ueber die Natur der menschlichen Sinnesempfindungen», Wissenschaftliche Abhandlungen, vol. 11, Leipzig, p. 591-609, cité par R. Blanché, La Méthode expérimentale et la philosophie de la physique, Paris, Colin, 1969, p. 195.

2. Cassirer en 1925 ne traite de la question que de manière incidente, en connexion avec la sémiotique de Helmholtz. R. Kahl, éditeur et traducteur des Selected Writings of Hermann von Helmholtz, (Middletown, Connecticut, 
dernier point qui fera l'objet d'une attention particulière dans la dernière partie de cet article. Nous comptons indiquer en premier lieu comment Helmholtz, à partir de ses investigations en physiologie, en vint à considérer les sensations en tant que signes. Nous exposerons ensuite cette conception sémiotique des sensations pour en dégager les implications sur le plan épistémologique. Nous discuterons enfin, tout en les situant dans le contexte historique plus large du développement de la problématique empiriste, certains aspects de la théorie helmholtzienne des sciences qui témoignent d'un point de vue fortement anti-réaliste. Néanmoins, le tracé de l'évolution des idées de Helmholtz sur la question indique, en fin d'analyse, que celui-ci aura toujours conservé une optique réaliste à l'égard des lois de la nature, adhérant ainsi à une forme de réalisme que l'on pourrait qualifier de réalisme nomologique.

I. On a souvent caractérisé l'époque de Helmholtz comme celle où l'on assistait en physique à une réfutation de la philosophie kantienne des sciences, alors qu'en fait, elle fut plutôt marquée par une transformation de celle-ci3. Plusieurs physiciens (dont Helmholtz) ressentaient en effet le besoin d'amender le kantisme dans lequel ils avaient été formés. Si les physiciens allemands de la génération antérieure avaient coutume d'aborder volontiers les questions philosophiques, ils avaient également été unanimes dans leur hostilité à l'endroit de la Naturphilosophie de

Wesleyan University Press, 1971, p. Xx), y consacre deux paragraphes pour conclure : "On the whole the [Helmholtz] continued to be a persistent realist, arguing, among other things, that a correct analysis of perception, while it does not provide conclusive evidence, does support the realist position."

3. On ne saurait en aucun cas parler d'un rejet de la philosophie kantienne des sciences. Dans son excellent chapitre sur l'arrière-plan intellectuel de l'empirisme logique, Suppe, dans The Structure of Scientific Theories, $\left(2^{\circ}\right.$ éd., Urbana, University of Illinois Press, 1977, p. 10), note d'ailleurs avec justesse : "By the turn of the century, the three main philosophic positions held in the German scientlic community were mechanistic materialism, neo-Kantianism, and Machian neo-positivism, with neo-Kantlanism being the most commonly held," 
l'idéalisme ambiant 4 . Helmholtz lui-même ne manqua pas de déplorer la relation malheureuse qui, sous l'influence de ce qu'il appelait la "philosophie de l'identité" de Hegel et Schelling, s'était instaurée entre la philosophie et les sciences de la nature. Parlant de cette génération antérieure, il remarquait que :

La philosophie de la nature de Hegel apparaissait, aux yeux des scientifiques à tout le moins, tout à fait dénuée de sens. Des nombreux scientifiques remarquables qui furent ses contemporains, il ne s'en trouva pas un seul qui se soit senti près de ses idées 5 .

En revanche, Helmholtz se réclama constamment de Kant. Dans la préface de la $3^{e}$ édition de Vortraege und Reden, Helmholtz écrit :

J'étais un kantien fidèle dès le début de ma carrière et je le suis toujours; ou plutôt, je croyais alors que les changements que je désirais apporter à la philosophie de Kant étaient d'im-

4. C. Jungnickel et R. McCommach, dans Intellectual Mastery of Nature, vol. 1 : Theoritical Physics from Ohm to Einstein, (Chicago, University of Chicago Press, 1986, p. 23-24), offrent un aperçu des manuels de physique de cette époque : "The move away from Aristotelian textbooks, which had occurred not long before, to textbooks that stressed experience had been followed by renewed attention to the nature of objective knowledge and particularly to the process by which experience becomes scientific knowledge." Ils ajoutent (p. 27) : “Except for matters of emphasis, the physicists quarrel was not with philosophy as a whole but only with a part of it, Naturphilosophie, which they often did not even dignify with the name of philosophy. The heated campaign of the physicists against the nature philosophers did not stop with denunciations in their textbooks. These were faint echoes of what they said in private letters, reviews, addresses, periodicals, and elsewhere.» Les auteurs soulignent par ailleurs l'influence exercée sur les physiciens par les Premiers fondements de la métaphysique des sciences de la nature de Kant.

5. (1862) “Ueber das Verhaeltnis der Naturwissenschaften zur Gesammtheit der Wissenschaft", Abhandlungen zu Philosophie und Naturwissenschaft, Darmstadt, Wissenschafliche Buchgesellschaft, 1966, p. 14. Sauf indication contraire, la traduction française des citations est de nous. 
portance secondaire et pouvaient être mis en retrait par rapport à ce que j'estime encore être son résultat fondamental ${ }^{6}$.

Pourtant, ce que Helmholtz amendait chez Kant pourrait difficilement être considéré comme une question d'importance secondaire : il s'agissait du statut épistémologique des postulats de la géométrie. Parvenant de façon indépendante et différente à des résultats obtenus peu avant par Riemann 7 , Helmholtz montra que la géométrie est basée sur certains faits et constitue donc une science empirique. Ultérieurement, Helmholtz admit que l'espace pouvait être considéré comme une forme à priori de l'intuition, dans la mesure où la structuration spatio-temporelle de nos perceptions est nécessaire et universelle; mais la question de savoir si cet espace est euclidien ou non (question qui est impliquée dans le choix des axiomes géométriques) demeurait bel et bien une question d'ordre empirique.

Helmholtz pouvait cependant affirmer de plein droit sa fidélité à Kant. Ses recherches sur les fondements de la géométrie contredisaient certes l'Esthétique transcendantale, mais il n'adhérait pas moins à un postulat fondamental de la théorie kantienne des sciences, à savoir que le principe de causalité est d'ordre transcendantal. C'est dire que la causalité n'est pas objet d'expérience : elle est plutôt ce qui rend l'expérience possible. Principe régulateur de la pensée, la loi de causalité cautionne la légalité ou régularité nomologique [Gesetzmaessigkeit] des phénomènes naturels et représente leur accessibilité à la pensée.

6. (1894) Einleitung zu den Vorlesungen ueber theoretische Physik, éds. A. Koenig et C. Runge, Leipzig, Barter, 1903, p. vii.

7. Helmholtz rédigea une série de six articles sur la géométrie entre 1866 et 1878. Les deux premiers, (1866) “Ueber die thatsaechlichen Grundlagen der Geometrie» et (1868) "Ueber die Thatsachen, die der Geometrie zum Grunde liegen», recoupent en plusieurs points essentiels le fameux article de Riemann "Ueber die Hypothesen, welche der Geometrie zum Grunde liegen» (lequel fut présenté comme thèse de doctorat en 1854 mais ne fut pas publié avant 1868). 
Si nous présupposons, dit Helmholtz, qu'il est possible de parachever notre compréhension des phénomènes naturels, c'està-dire d'exhiber quelque chose d'ultime et d'inchangeant qui soit la cause des changements que nous observons, alors nous devons accepter un principe régulateur de la pensée. $\mathrm{Ce}$ principe est la loi de la causalité et il exprime notre croyance en l'entière intelligibilité du monde. La compréhension, poursuit-il, est la méthode par laquelle la pensée acquiert la maîtrise du monde, ordonne les faits et détermine le futur à l'avance. C'est son droit et son devoir d'étendre l'application de cette méthode à tout ce qui se produit, et en cela, elle a déjà obtenu effectivement des succès considérables. Mais nous n'avons d'autres garanties de l'applicabilité du principe de causalité que celles de son succès [...]. En fait, de préciser Helmholtz, la loi de causalité est en réalité une loi transcendantale, une loi qui est donnée à priori. II est impossible de l'établir par voie empirique $[. . .]^{8}$.

II. Le caractère transcendantal du principe de causalité sert de point de départ à la théorie de la perception de Helmholtz, théorie qu'il conçoit, d'une part, comme le parachèvement scientifique de la philosophie kantienne et, d'autre part, comme l'extension épistémologique de ses propres investigations en physiologie optique et acoustique.

On sait que Kant avait distingué deux facultés de représentation: l'une, purement réceptive, la sensibilité, et l'autre, qu'il qualifiait de "spontanée», l'entendement. Ces deux fonctions mutuellement exclusives devaient jouer un rôle complémentaire dans la constitution d'une connaissance qui ait une valeur objective. Kant appelait «synthèse" l'activité fondamentale de l'entendement, processus dans lequel «une multiplicité de représentations sont réunies sous une seule". On pouvait alors lire dans l'Analytique transcendantale comment l'entendement agit déjà

8. (1878) "Die Tatsachen in der Wahrnehmung", Die Tatsachen in der Wahrnehmung/Zaehlen und Messen, Darmstadt, Wissenschaftliche Buchgesellschaft, 1959, p. 47. 
dans la formation de nos perceptions, en ce que celles-ci sont le produit d'une synthèse de sensations multiples (les sensations constituant le donné brut dans la sensibilité). Helmholtz adopta le même point de vue tout en mettant l'accent sur la dimension causale de la perception :

Ce qui est sans équivoque et ce que nous retrouvons comme fait libre de toute hypothèse, c'est la légalité du monde phénoménal. D'emblée, la perception simple d'objets stationnaires distribués dans l'espace comporte la reconnaissance d'un lien causal entre nos mouvements et les sensations qui en résultent. Les représentations les plus élémentaires contiennent donc déjà en elles-mêmes un élément de pensée et s'accomplissent en accord avec les lois de la pensée. Tout ce qui, dans l'intuition, s'ajoute au matériel brut des sensations, s'analyse en tant que pensée $[. . .]^{9}$.

Helmholtz accorda cependant une meilleure part à l'expérience et à certaines activités inconscientes de l'esprit dans le processus de la perception. II reprit à son compte les thèses de son ancien professeur de Berlin, Johannes Mueller, qui avait établi le principe des énergies spécifiques des fibres nerveuses.

À l'instar de Mueller, Helmholtz postule d'emblée l'existence d'une relation de cause à effet entre les objets extérieurs et nos sensations. Celles-ci sont causées par l'excitation que provoquent les objets extérieurs sur nos organes sensoriels. Ces excitations sont transmises au cerveau et c'est dans le cerveau qu'elles deviennent des sensations conscientes qui sont combinées les unes avec les autres et donnent lieu à ce nous appelons des perceptions. Dans le cas de l'œil, par exemple, les perceptions visuelles sont produites non pas directement sur chaque rétine, mais dans le cerveau; c'est ainsi que Helmholtz peut dire que «tout ce qui, dans l'intuition, s'ajoute au matériel brut des sensations, s'analyse en tant que pensée».

9. Ibid., p. 42. 
Les sensations causées par l'action des objets extérieurs se divisent en cinq groupes correspondant aux cinq sens, et les différences entre elles sont telles qu'il est impossible de comparer les sensations appartenant à différents groupes (comparer une sensation de bleu avec une sensation d'acidité, par exemple) ou de passer d'un groupe à l'autre, comme c'est le cas des sensations à l'intérieur d'un même groupe (où une sensation de bleu peut très bien passer à une sensation de vert, par exemple). Helmholtz appelle cette différence une différence de modalité. Les sensations sont toujours groupées à l'intérieur d'une même sphère qualitative (Qualitaetskreis) du sens en question. L'excitation d'un nerf peut produire, par exemple, un mouvement, une sécrétion ou une sensation, selon que l'on excite un nerf moteur, un nerf glandulaire ou un nerf sensoriel. Une sensation produite lorsqu'un nerf est excité aura nécessairement la qualité du sens dont le nerf excité sert à transmettre l'influx. Ainsi, une excitation du nerf optique produit uniquement des sensations de lumière, que le nerf optique soit excité par un rayon lumineux, par un coup sur le globe oculaire, ou par une tension exercée sur le nerf provenant d'un mouvement rapide de l'œil. Si une même sensation peut être causée par différents stimuli, un même stimulus extérieur, d'autre part, produira différentes sortes de sensations selon qu'il excite différentes sortes de nerfs :

Le même courant électrique dont la présence nous est indiquée par le nerf optique en tant que jet de lumière, ou par l'organe du goût en tant que saveur acide, excite dans le nerf de la peau la sensation d'un brûlement. Le même rayon de soleil, qui est appelé lumière lorsqu'il tombe sur l'œil, nous l'appelons chaleur lorsqu'il tombe sur la peau ${ }^{10}$.

10. (1853) "Ueber Goethes naturwissenschaftliche Arbeiten", Abhandlungen zu Philosophie und Naturwissenschaft, Darmstadt, Wissenschaftliche Buchgesellschaft, 1966, p. 113. 
Helmholtz endosse donc le principe de Mueller selon lequel les "énergies" qui sont les causes de nos sensations ne sont pas immanentes aux objets extérieurs, mais relèvent plutôt de la composition physiologique de nos organes sensoriels. C'est dire que les différentes qualités perçues par les sens sont uniquement les effets spécifiques des organes en question, quelle que soit la nature de l'objet extérieur. En termes kantiens, il y a en effet des conditions universelles et nécessaires à ce qui se donne dans les sens, mais ces conditions sont déterminées par la constitution physiologique des sens visuel, tactile, auditif, etc. C'est ainsi que la loi des énergies nerveuses spécifiques de Mueller procure, aux yeux de Helmholtz, une assise empirique à ce que Kant avait tenté d'établir de façon à priori.

III. Ces investigations physiologiques démontrent l'absence d'un lien de similitude entre nos sensations et les objets extérieurs, la qualité de nos sensations ne reflétant aucunement la nature des objets qui les produisent. Quel lien existe-t-il alors ? C'est ici qu'entre en jeu la conception sémiotique des sensations. Que nos sensations soient causées par les objets extérieurs sans toutefois leur correspondre qualitativement (la cause étant différente de l'effet) ne pouvait signifier pour Helmholtz qu'une chose : nos sensations ne sont que des signes des objets.

Nos sensations sont justement des effets produits sur nos organes par des causes extérieures; évidemment, la façon dont ces effets se manifestent dépend essentiellement du type d'appareil sur lequel ils se produisent. Dans la mesure où la qualité de nos sensations nous informe des caractéristiques des causes extérieures qui les produisent, elles peuvent être considérées comme des signes des objets extérieurs, mais non comme des images. Car on exige d'une image qu'elle possède une certaine similitude avec l'objet représenté, d'une statue une similitude de forme, d'un dessin une similitude de projection perspective dans le champ visuel, d'une peinture encore une similitude de couleurs. Un signe, par contre, ne demande aucune sorte de ressemblance avec ce dont il est le signe. La 
relation de l'un à l'autre se limite à ce que le même objet, affectant les sens sous les mêmes conditions, produit toujours le même signe ${ }^{11}$.

Tous les signes sont soumis à l'interprétation. Nos perceptions, c'est-à-dire les idées que nous avons des objets, résultent d'une interprétation des sensations qui s'accomplit à un niveau inconscient. Plus précisément, cette interprétation est assurée par des inférences inconscientes ${ }^{12}$ où œuvre déjà, comme nous l'avons vu, le principe de causalité. Ces inférences sont induites par d'incessants mouvements et sentiments de mouvement de l'agent percevant. De façon générale, les perceptions proviennent essentiellement de l'activité inconsciente de l'esprit qui compare et associe une grande quantité de signes, apprenant ainsi à les utiliser comme on apprend à utiliser les mots ${ }^{13}$. Elles sont le produit d'un processus d'interprétation qui débute à la naissance et qui continue tout au long de la vie. La perception s'apprend et cet apprentissage se conçoit pour Helmholtz de façon analogue à l'apprentissage de la langue maternelle (dont on dit qu'elle est acquise de façon inconsciente, que sa grammaire est, selon

11. "Die Tatsachen...", p. 18-19.

12. Helmholtz parle de "conclusions inconscientes", qui sont le produit d'actes de jugement inductifs. Nous croyons à la réalité subphénoménale, mais cette réalité ne sera toujours que réalité inférée, avec toute l'incertitude attribuée aux jugements inductifs.

13. Ainsi, par exemple, le simple phénomène de la constance des couleurs (alors que la luminosité varie) signifiait pour Helmholtz que la perception des couleurs ne pouvait se réduire à un processus mécanique de transposition de longueurs d'ondes en couleurs, l'organisme et le cerveau devant jouer un rôle actif dans le phénomène de la perception. En cela, Helmholtz se dégageait de la tradition naturaliste de Newton et de Young à laquelle il appartenait. Quant aux «actes de jugement" inhérents à la perception visuelle (comme à toute perception), la conception d'alors du système nerveux en tant que mécanisme statique plutôt que processeur dynamique, empêchait de voir en quoi ils pouvaient bien consister. Les travaux fondamentaux de Marr, de Land et Zeki, en particulier leur modèle computationnel de la perception, se situent à cet égard dans la tradition initiée par Helmholtz. 
le terme chomskien, «internalisée»). Ces systèmes sémiotiques diffèrent essentiellement par le fait que les sensations, par opposition aux signes linguistiques, sont des signes naturels ${ }^{14}$.

IV. Que nos sensations soient uniquement des signes, et non des images des choses, peut sembler mettre en question notre pouvoir de nous former une image de la réalité, ou à tout le moins une image objective de la réalité. Mais le temps, allié au principe de causalité, fait bien les choses. Puisqu'il y a un lien de causalité entre le signe/ sensation et l'objet qui le cause et puisque le principe de causalité nous assure que sous des conditions identiques, les mêmes causes produisent les mêmes effets, nous savons que dans ces conditions, différents signes, en tant qu'effets, renvoient toujours à différentes causes. Cela semble peu, mais c'est beaucoup :

À l'opinion populaire qui prête foi à l'entière véracité des images que nos sens nous livrent des choses, ce résidu de ressemblance que nous sommes prêts à reconnaître peut sembler trivial. En vérité il ne l'est pas, car il nous livre quelque chose de la plus grande importance : il nous livre une image de la légalité des événements du monde réel ${ }^{15}$.

De fait, ce n'est que sous l'aspect temporel que Helmholtz voyait une correspondance déterminée entre nos perceptions et le monde extérieur : la succession de nos sensations est une reproduction fidèle de la séquence

14. "La différence essentielle entre la symbolique du langage humain et cette symbolique de nos nerfs sensoriels me paraît être que celle-là est une œuvre arbitraire de la volonté, tandis que celle-ci nous est donnée par la nature ellemême, qui a constitué nos corps d'une manière déterminée. La langue de nos nerfs sensoriels ne comporte pas de familles ni de dialectes, elle est la même pour toute l'humanité, et c'est pourquoi nous devons supposer chez chaque homme, à l'exception de quelques écarts pathologiques, l'intelligence de nos propres sensations." Helmholtz, «Ueber die Natur...», cité par R. Blanché, La Méthode..., p. 195.

15. Helmholtz, “Die Tatsachen...», p. 19. 
temporelle des événements qui les causent. De plus, le caractère bi-univoque du lien qui unit les événements avec les sensations/signes qu'ils produisent fait en sorte que les relations nomologiques existant entre les événements seront reproduites par des relations nomologiques entre les sensations :

Puisque les mêmes choses dans le monde de l'expérience sont indiquées par les mêmes signes, à la suite régulière des mêmes causes produisant les mêmes effets correspondra une suite régulière dans le domaine de nos sensations ${ }^{16}$.

La relation causale qui existe entre l'objet extérieur et la sensation nous assure donc que sous des conditions identiques de l'objet extérieur et de l'observateur, les mêmes sensations sont produites, de sorte que la régularité que nous découvrons dans la séquence temporelle de nos sensations est une image, et non simplement un signe, de la régularité nomologique des événements qui les causent. Si nous ne pouvons pas savoir à quoi correspondent nos sensations dans l'ordre des choses, en revanche, le principe de causalité nous assure que toute différence dans l'ordre des signes sensoriels correspond à une différence dans l'ordre des choses ${ }^{17}$. Nous pouvons nous faire une image objective de la réalité, mais celle-ci n'est objective qu'en tant qu'image des changements et des lois qui régissent ces changements ${ }^{18}$.

\section{Ibid.}

17. Les sensations ont à peu près le même rapport avec le monde réel que le nom d'une personne ou la graphie de ce nom avec cette personne elle-même : "En nous apparaissant comme identiques ou comme différentes, ce qu'elles nous apprennent, c'est si nous avons ou non affaire aux mêmes objets et aux mêmes propriétés du monde réel [...]. Mais elles ne nous apportent rien de plus.» Ibid., p. 194-195.

18. Ces idées seront reprises avec force par Heinrich Hertz dans son introduction aux Principes de mécanique : “Nous nous faisons des images artificielles internes [innere Scheinbilder] ou des symboles des objets extérieurs, et la forme 
Comme le remarque U. Mayer ${ }^{19}$, Helmholtz aura le premier résolu la question de savoir ce qu'une image doit avoir en commun avec ce qu'elle représente lorsque les éléments de l'image et les éléments représentés sont de nature différente. En termes contemporains, on pourrait dire que la relation entre la séquence des sensations internes et celle de leurs causes extérieures est une relation d'identité structurelle ou d'isomorphie. C'est en raison de cette isomorphie que nous sommes en mesure de nous représenter la régularité nomologique des événements du monde extérieur :

Je n'ai pas à discuter le fait qu'il est contradictoire de vouloir se représenter le réel ou la chose en soi de Kant à l'aide de déterminations directes qui ne tiennent pas compte de la forme de notre représentation. Ceci a souvent été exposé. Ce que nous pouvons atteindre cependant, c'est la connaissance de l'ordre légal dans le domaine du réel, cet ordre étant représenté dans le système de signes que constituent nos impressions sensorielles ${ }^{20}$.

Helmholtz est donc à l'origine d'une conception structuraliste qui lui fournit une explication de la possibilité de la mécanique newtonienne comme description des mouvements de la matière dans l'espace. Soulignons en plus que

que nous leur donnons est telle que les rapports logiques [denknotwendige Folgen] entre les images sont en retour une image des rapports nomologiques [naturnotwendige Folgen] entre les objets représentés. [...]. Les images dont nous parlons sont nos représentations des choses; elles possèdent avec les choses cette conformité essentielle qui découle de la conformité entre notre esprit et la nature; mais point n'est besoin qu'elles possèdent quelque autre conformité aux choses. En effet nous ne savons pas et nous n'avons aucun moyen de savoir à quoi peuvent correspondre nos représentations, sauf justement pour ce rapport fondamental." Die Prinzipien der Mechanik, Gesammelte Werke, vol. 111, Lepzig, 1894, p. 1-2.

19. Semantischer Konventionalismus. Habilitationsschrift, Goettingen, p. 11-24.

20. “Die Tatsachen...", p. 45. 
la théorie helmholtzienne de la science a ceci de particulier qu'elle fait reposer la connaissance du monde extérieur sur un processus fondamental unique, la formation de symboles, et non pas, comme c'était le cas chez Kant, sur deux processus mutuellement exclusifs d'intuition et d'intellection.

V. Ce recours de Helmholtz à une conception sémiotique des sensations est au cœur d'une prise de position complexe face à la question du réalisme en science ${ }^{21}$. Contrairement à l'opinion voulant que Helmholtz aurait toujours professé un point de vue réaliste, bien que chancelant, tout particulièrement dans son Introduction aux cours de physique théorique de 1894, nous voulons soutenir que Helmholtz aura adopté dès le début une position plus nuancée sur la question. Déjà dans l'introduction du Mémoire sur la conservation de la force de 1847, qui est son premier article portant sur la physique, Helmholtz apporte des considérations qui mettent directement en cause le point de vue réaliste. Ces mêmes considérations seront reprises et développées dans le troisième tome du Traité de physiologie en 1867, ainsi que dans "Les faits dans la perception" en 1878, un article majeur qui résume les résultats de ses investigations en physiologie, en dégage les implications philosophiques et révèle des traits anti-réalistes accentués. Ces aspects anti-réalistes de la théorie des sciences de Helmholtz réapparaîtront de façon plus systématique dans l'Introduction aux Cours de physique théorique.

21. La position de Helmholtz est certes plus complexe que ne le laisse entrevoir le passage de Kahl cité au tout début. Celui-ci ressent d'ailleurs le besoin d'ajouter : "Sometimes, especially in the Introduction to the Lectures on Theoretical Physics, his [Helmholtz] scientific realism faltered for a moment and he favored the position that to use abstract substantives such as force was only a façon de parler. It was convenient and consistent with the natural, common-sense realism of everyday life; but a formulation, for example, of a law of motion using the term force ("the force of gravityw) was, he maintained at these times, empirically no more than a description of motion." Selected..., p. XX. 
V.1 Le premier de ces aspects concerne la notion de force et le statut ontologique accordé aux forces dont traite la mécanique. S'il est caractéristique de la position réaliste d'accorder une réalité objective à ces différentes forces, il semble bien que le point de vue de Helmholtz s'en détache de façon marquée.

L'introduction du Mémoire débute par des considérations générales concernant le but de la science. Le but de la physique, nous dit Helmholtz, est de découvrir des lois qui puissent lier les phénomènes naturels à des principes généraux et les déduire de ceux-ci. La découverte de ces lois est la tâche de la physique expérimentale, alors que la physique théorique cherche à trouver les causes des phénomènes sur la base de leurs effets observables. Cette recherche des causes est justifiée et imposée par le principe voulant que tout changement dans la nature ait une cause suffisante. Les causes que nous attribuons aux phénomènes peuvent être invariables ou variables; dans le second cas, le même principe qui nous conduit à chercher les causes de tout changement nous incitera à chercher de nouveau les causes de cette variation, de sorte que ce n'est que par la découverte de causes constantes que la compréhension ultime des phénomènes naturels sera pleinement atteinte. Le Mémoire insiste sur le fait que pour satisfaire à cette exigence, la physique doit ramener tous les phénomènes naturels à des forces attractives ou répulsives invariables, à des forces motrices centrales dont l'intensité ne dépend que des rapports de position dans l'espace.

La science, de continuer Helmholtz, a recours à deux abstractions dans sa conceptualisation des objets du monde extérieur. Nous formons la notion de matière lorsque nous considérons les objets extérieurs sous le simple aspect de leur existence, indépendamment des effets qu'ils produisent sur les autres objets ou sur nos organes sensoriels. Prise en soi, la matière est inaltérable et inaltérante. La masse (comme quantité fixe de matière) et l'étendue 
sont ses seuls attributs; les différences qualitatives ne peuvent résider dans la matière en tant que telle, mais uniquement dans les effets qu'elle produit. Nous n'avons la connaissance des objets extérieurs que par l'action qu'ils exercent sur nos organes sensoriels; c'est sur la base de cette action que nous inférons l'existence d'un agent et que nous pensons l'objet extérieur. La matière pure est donc une impossibilité conceptuelle et notre compréhension de la nature exige la formation d'une seconde abstraction, la notion de force en tant que capacité de produire un effet. D'autres passages du Mémoire définissent les forces comme les lois des effets que les objets extérieurs produisent sur les autres objets ou sur nos organes. Ces deux abstractions sont pour Helmholtz des notions corrélatives :

II est certain que les idées de matière et de force sont réellement inséparables. La matière pure serait indifférente à tout le reste du monde, puisqu'elle ne modifierait aucun objet voisin et n'affecterait pas non plus nos organes; et si la force pure existait, elle ne serait que ce que nous avons déjà nommé matière ${ }^{22}$.

Dans un appendice ajouté au Mémoire en 1881, Helmholtz précise que l'impossibilité de concevoir les deux notions prises isolément découle simplement du fait que la loi d'un effet (c'est-à-dire une force) présuppose certaines conditions sous lesquelles elle est réalisée : "Une force séparée de la matière serait l'objectivation d'une loi, à laquelle il manque les conditions de sa réalisation ${ }^{23}$ ". Penser une force, c'est-à-dire une capacité de produire des effets, indépendamment des effets qu'elle produit, est vide de

22. (1847) "Ueber die Erhaltung der Kraft», Wissenschaftliche Abhandlungen, vol. 1 (1882), p. 12-75. Trad. fr. sous forme de monographie Mémoire sur la conservation de la force, Paris, Masson, 1869. Également sous forme de monographie, Ueber die Erhaltung der Kraft, Weinheim, Physik - Verlag, 1983.

23. Ibid., p. 68. 
sens. Ce principe est à l'origine d'une tradition en physique qui s'interdira de parler de forces en termes généraux, sans la donnée des conditions sous lesquelles elles sont réalisées. Ce même principe fonde la méfiance à l'égard des diverses «forces» dont traite la mécanique pour expliquer les phénomènes naturels et il sert d'appui au point de vue descriptiviste dont Kirchhoff (qui avait rejoint Helmholtz à Berlin en 1874) fut le représentant le plus influent ${ }^{24}$. S'il n'adhère pas entièrement au descriptivisme strict de Kirchhoff et s'il considère notamment les notions de cause et de force comme essentielles à la compréhension des phénomènes naturels et parties intégrales de l'explication scientifique, Helmholtz ne les subordonne pas moins à la notion de loi. Nous parlons de causes lorsque nous reconnaissons les lois comme indépendantes de notre pensée, et nous parlons de forces lorsque nous les reconnaissons comme indépendantes de notre volonté, c'est-à-dire comme réelles.

24. Vorlesungen ueber mathematische Physik, Mechanik, $2^{\ominus}$ éd., Leipzig, Teubner, 1877, préface : "On définit usuellement la Mécanique comme la science des forces, et les forces, comme les causes qui produisent les mouvements ou ont la capacité de les produire. [...] [Cette définition] est cependant entachée de l'obscurité inhérente aux concepts de cause et de capacité. [...] Eu égard à la rigueur des démonstrations que la Mécanique permet par ailleurs, il m'est apparu désirable d'en écarter de telles obscurités, même si cela ne s'avère possible qu'en restreignant la tâche qu'elle doit se donner. C'est ainsi que je donne pour tâche à la Mécanique de décrire les mouvements qui se produisent dans la nature, d'une façon complète et qui soit la plus simple possible. J'entends par là qu'il s'agit uniquement pour la Mécanique de déterminer quels sont les phénomènes qui se produisent, et non d'en rechercher les causes. Partant de ce principe et supposant les représentations d'espace, de temps et de matière, on parvient par des moyens purement mathématiques à formuler les équations générales de la Mécanique. Ce faisant, on introduit le concept de force sans être en mesure d'en donner une définition complète. L'incomplétude de cette définition n'entraîne ici aucune obscurité, car l'introduction de forces ne constitue qu'un moyen de simplifier la formulation de la théorie, en l'occurence : d'exprimer en peu de mots des équations qui, sans l'aide de ce terme, ne pourraient être qu'alourdies. II suffit ici pour écarter toute obscurité de donner une définition du terme qui fasse en sorte que tout énoncé de la Mécanique où il est question de forces puisse être traduit en équations." 
D'autre part, ajoute-t-il, il est clair qu'on ne peut distinguer entre la pensée et la réalité tant qu'on n'est pas en mesure de savoir ce qu'il appartient au moi de pouvoir changer ou non. Ceci, en retour, n'est possible que lorsque nous sommes à même de reconnaître les effets uniformes de nos actes de volonté. De sorte que le fait d'avoir caractère de loi est le présupposé essentiel à ce qui a caractère de réalité 25 .

Somme toute, Helmholtz professe un réalisme des causes parce qu'il juge que la catégorie de la causalité est nécessaire à l'intelligibité du phénomène en tant que phénomène; d'autre part, ce réalisme est un réalisme critique dans la mesure où notre connaissance des causes est toujours hypothétique et sujette à révision. Quant aux forces, elles sont réelles dans la mesure où elles sont effectives (l'effectivité [Wirklichkeit] étant le terme allemand pour désigner ce qui est réel). Pour Helmholtz, la notion générale de force aura toujours constitué une abstraction. S'il est vrai que le Helmholtz du Mémoire considère les forces centrales comme objectives, il n'en demeure pas moins que la réalité des forces se résume pour lui à la réalité des lois. II faut cependant noter que ce réalisme se situe sur le plan métaphysique et non sur le plan scientifique, car c'est bien le règne des lois (et non les lois) qui, pour Helmholtz, est exempt de tout caractère hypothétique.

Le scepticisme de Helmholtz par rapport à l'objectivité des forces dont traite la mécanique sera nettement accentué dans l'Introduction aux cours de physique théorique, où il se rapproche considérablement de la conception descriptiviste citée plus haut. Se réclamant de la tradition des Faraday (auquel il vouait une grande admiration), Maxwell et Kelvin, et reprenant les termes mêmes de Kirchhoff, il déclare que les diverses lois du mouvement ne constituent rien de plus qu'une description des événements observables, tout en ajoutant qu'une description «complète et la

25. “Die Tatsachen...», p. 45. 
plus simple possible" des phénomènes naturels ne va pas sans la formulation de lois impliquant des forces (lois dynamiques). Helmholtz indique cependant qu'il préfère parler de "lois dynamiques" plutôt que de "forces", car du point de vue descriptiviste, la notion de force, en tant que substance, n'a aucun contenu factuel. «En fait, dit-il, nous ne désignons rien de plus par le concept de force, à tout le moins rien qui ajoute au sens factuel contenu dans la simple description du phénomène $[. . .]^{26}$.» Un peu plus loin dans cette section, Helmholtz affirme que "parler de la force, en tant qu'agent, ne désigne rien de plus sur le plan factuel et n'a d'autre contenu réel que celui de vouloir exprimer que la loi se manifestera à chaque fois que les conditions de sa manifestation seront données ${ }^{27}$ ". Ce point de vue instrumentaliste qui ne voit la nécessité de la notion de force que dans le contexte de la prédiction ou dans l'optique de l'économie de la description théorique ne fait pas qu'anticiper la pensée d'un Hertz ou d'un Mach, il préfigure également toute la problématique empiriste logique des "termes auxiliaires" et de leur éliminabilité (syntaxique et sémantique) 28 . L'idée qu'une force (c'est-à-dire une capacité de produire des effets) devienne vide de sens lorsque conçue en tant que substance (c'est-à-dire pensée indépendamment des effets qu'elle produit) trouve donc sa formulation définitive dans la thèse à l'effet que la force, séparée de ses conditions de réalisation, ne possède pas de "contenu factuel». L'empirisme logique devra également reprendre cette dernière notion pour la préciser en tant que notion métathéorique et la généraliser au niveau

26. Einleitung..., p. 11.

27. Ibid., p. 15

28. Nous faisons ici référence à l'utilisation épistémologique qu'ont fait Carnap et Hempel des résultats de W. Craig et de F.P. Ramsey portant sur la définissabilité des termes qui servent à l'extension lexicale d'un système axiomatique formel du premier ordre. 
de la théorie entière ${ }^{29}$. Il est intéressant de noter que Helmholtz considérait déjà la notion de "contenu factuel» ou de "contenu empirique" comme une notion sémantique («le sens factuel»).

L'Introduction aux cours de physique théorique fait intervenir un élément nouveau qui permet à Helmholtz de préciser de façon systématique sa pensée sur le statut ontologique qu'on doit accorder aux forces de la mécanique. Le caractère systématique de sa prise de position anti-réaliste provient du fait qu'il l'incorpore maintenant à des considérations portant sur le langage. Dans la même première section de l'ouvrage, Helmholtz dit que nous sommes naturellement portés à passer du mode d'expression verbal au mode d'expression substantif; de sorte que plutôt que de formuler, par exemple, la loi de gravitation, nous sommes amenés à parler de la force de gravitation, laquelle, en fait, ne fait que désigner sur le mode substantif ce que la loi dynamique décrit sur le mode verbal. C'est ainsi qu'il écrit :

En règle générale, l'expression linguistique [des lois du mouvement] dévie des formulations que nous en avons données jusqu'ici, en ce que nous formons des abstractions et nous employons des substantifs à la place de verbes; c'est ce qui arrive, par exemple, lorsque nous exprimons la première des lois citées plus haut en disant qu'il existe une force d'attraction constante d'une grandeur déterminée entre deux corps séparés l'un de l'autre d'une distance finie dans l'espace. Au lieu d'une simple description du phénomène du mouvement, nous introduisons ainsi une entité abstraite, la force d'attraction [...]. Cette façon de parler a suscité maintes objections [...]; elle a souvent été interprétée comme impliquant que ce substantif abstrait, la force, désigne quelque chose qui existe essentiellement, et comme justifiant l'énoncé de certaines thèses sur les propriétés

29. En l'occurrence, le contenu factuel d'une théorie physique sera identifié à l'ensemble des conséquences logiques observables des axiomes de la théorie, considérée comme système axiomatique formel. Un ensemble ouvert de crègles de correspondance" établit la mise en correspondance du système formel avec des systèmes physiques observables. 
essentielles des forces, lesquelles, si elles sont exactes, ne sont cependant soit que des tautologies, soit que des thèses qui n'ont que l'apparence de posséder un contenu réel ${ }^{30}$.

Plus loin, il note que nous "désignons habituellement les choses - celles qui perdurent dans leur existence, qui manifestent une certaine puissance et qui déterminent le monde extérieur sans que nous ayons à intervenir - par des substantifs que nous employons normalement pour des choses qui existent réellement" et il ajoute :

\begin{abstract}
C'est manifestement la raison fondamentale qui, plutôt que de parler de lois de force, nous fait adopter le mode substantif comme mode d'expression préféré [...]. De ce substantif hypothétique, et c'est ainsi que nous devons concevoir une force, nous ne savons rien de plus qu'il est dans son essence de produire certains effets ${ }^{31}$.
\end{abstract}

On retrouve donc l'idée que la notion de "force" n'est ni plus ni moins que le fait d'une substantivation de la notion de "loi de force", substantivation qui nous incline au réalisme scientifique. Cette explication du point de vue réaliste comme émanant d'un fait de langage ("adopter le mode substantif comme mode d'expression préféré") anticipe la distinction de Carnap ${ }^{32}$ entre le mode matériel [inhaltlich] et le mode formel de parler : les thèses d'ordre ontologique sont en fait des énoncés métalinguistiques qui portent sur le cadre linguistique à adopter pour décrire un univers du discours donné. Cette résolution formelle du débat sur le réalisme et l'anti-réalisme sera plus tard géné-

30. Einleitung..., p. 11.

31. Ibid., p. 15.

32. Voir R. Carnap, "On the character of philosophical problems", Philosophy of Science 1 (1934), p. 5-19 et Logische Syntax der Sprache, Vienne, Springer, 1934. 
ralement reprise par les empiristes logiques ${ }^{33}$. Helmholtz considérait déjà que la thèse du réalisme scientifique ne peut s'exprimer que sur le mode matériel de parler; elle est cependant externe à la science, car les énoncés des lois de la physique (et cela est manifeste lorsqu'ils sont formulés sur le mode formel) n'ont aucune portée ontologique, c'est-à-dire qu'il ne comportent aucun présupposé sur la nature des objets du domaine du discours ${ }^{34}$.

V.Il Cette discussion de la notion de force nous aura donc permis de voir les éléments de la théorie helmholtzienne des sciences qui vont à l'encontre du réalisme scientifique et qui, d'autre part, préfigurent le développement ultérieur de la problématique empiriste. Nous voulons, en guise de conclusion, soulever quelques points d'ordre plus général qui complètent notre argumentation en faveur d'une réévaluation du réalisme imputé à Helmholtz.

33. L'ouvrage classique de E. Nagel, The Structure of science, $\left(2^{\theta}\right.$ éd., Indiapolis, Hackett, 1979), l'illustre de façon typique. Résumant le débat entre le réalisme et l'instrumentalisme (comme forme d'anti-réalisme) et reprenant la thèse de Carnap, Nagel conclut (p. 152) : aln brief, the opposition between these views is a conflict over preferred modes of speech.»

34. Lorsqu'il rédige l'appendice aux «Faits de la perception», Helmholtz a déjà incorporé sa prise de position sur le sujet à une philosophie du langage : «Dans cette première section je vais d'abord m'en tenir à l'hypothèse réaliste et en utiliser le langage, donc supposer que les choses que nous percevons objectivement existent en réalité et agissent sur nos sens. J'adopte cette voie dans l'unique but de pouvoir utiliser en premier lieu le langage simple et compréhensible de la vie quotidienne et de la science, pour ainsi m'expliquer sur ce que j'entends de la façon la plus compréhensible possible. Je me réserve de laisser tomber l'hypothèse réaliste dans les paragraphes ultérieurs et de reprendre la même discussion en langage abstrait, sans aucune présupposition quant à la nature du réel», "Die Tatsachen...», p. 61-62. Et plus loin : "L'hypothèse de l'idéalisme subjectif serait tout aussi admissible que le point de vue réaliste dont nous avons jusqu'ici utilisé le langage. Nous pouvons supposer que toutes nos perceptions ne sont qu'un songe, ne serait-ce qu'un songe hautement cohérent, où nos représentations se succèdent selon des lois déterminées. [...] Ce que nous appellons dans l'hypothèse réaliste des lois de la nature, mais dans l'hypothèse idéaliste des lois qui régissent la succession des représentations qui ont le caractère de perceptions." Ibid. p. 69-70. 
Si on est en droit d'exiger d'un tenant du réalisme qu'il n'épouse pas également la thèse adverse, alors force est d'admettre que Helmholtz n'est pas un tenant de la thèse réaliste. Comme nous venons de le noter ( $\left.n^{\circ} 34\right)$, Helmholtz considérait la thèse de l'idéalisme subjectif comme tout aussi admissible que celle du réalisme. Elles sont pour lui également possibles, également irréfutables, également métaphysiques :

[L'hypothèse réaliste] est sans aucun doute l'hypothèse la plus simple que nous puissions formuler; cependant, nous ne pouvons la considérer comme nécessairement vraie, car à ses côtés il y a d'autres hypothèses idéalistes tout aussi possibles et tout aussi irréfutables. II est bon de ne jamais perdre cela de vue pour éviter de tirer des faits plus qu'il ne s'en laisse tirer. Les différentes versions de l'idéalisme et du réalisme sont des thèses métaphysiques qui, tant qu'elles sont reconnues comme telles, possèdent leur entière justification scientifique, tout aussi dommageables qu'elles puissent devenir lorsqu'elles sont élevées au rang de dogmes ou de soi-disant nécessités de la pensée ${ }^{35}$.

C'est en raison de leur valeur heuristique que Helmholtz reconnaît aux hypothèses métaphysiques leur justification scientifique. Toutefois, les principes nettement empiristes d'explication scientifique qu'il adopte dans ses' propres travaux feront contrepoids à sa tolérance à l'égard de la métaphysique. Le traité d'Optique physiologique, qui se clôt sur un chapitre traitant des "principes des explications empiristes", en témoigne de façon éloquente :

J'accorde que nous sommes bien loin de connaître les phénomènes psychiques d'une manière rigoureusement exacte. Chacun peut, suivant la tendance spéculative à laquelle il accorde la préférence, nier absolument, comme les spiritualistes, ou admettre absolument, comme les matérialistes, la possibilité de pénétrer dans la nature de ces phénomènes. Mais le natu-

35. "Die Tatsachen...", p. 41-42. 
raliste, qui doit s'en tenir aux faits et à la recherche des lois qui les régissent, n'a pas à décider cette question. II ne faut pas oublier que le matérialisme est une spéculation ou une hypothèse métaphysique tout aussi bien que le spiritualisme; aussi lui refuserons-nous le droit de s'immiscer dans l'explication des faits naturels, avec des raisonnements à priori, sans s'appuyer sur des faits [...]. Quant à moi, je crois que c'est suivre une voie plus sûre que de rattacher l'explication des phénomènes de la vision à d'autres phénomènes [... $]^{36}$.

C'est dire que la thèse réaliste, en tant qu'hypothèse métaphysique, trouve difficilement droit de cité dans le modèle empiriste d'explication scientifique défendu par Helmholtz.

Soulevons enfin un dernier point qui touche cette foisci à la notion de vérité. II est généralement reconnu que la notion de vérité comme correspondance aux faits possède une forte connotation réaliste ou, à tout le moins, que l'application de cette notion aux théories scientifiques représente une forme (sémantique) de réalisme scientifique. Or, il est significatif que dans l'esprit de Helmholtz, la conception sémiotique des sensations récuse essentiellement la notion réaliste de vérité-correspondance au profit d'un conception pragmatique de la vérité :

Je crois donc que cela n'a absolument aucun sens, de parler d'une vérité de nos représentations autre qu'une vérité pratique. Les représentations que nous formons des choses ne peuvent être que des symboles, des signes naturels des objets, dont nous apprenons à nous servir pour régler nos mouvements et nos actions. Lorsque nous avons appris à déchiffrer correctement ces symboles nous sommes à même, avec leur aide, de diriger nos actions de façon à produire le résultat désiré, c'est-à-dire à faire naître les sensations nouvelles que nous attendons ${ }^{37}$.

36. (1867) Handbuch der physiologischen Optik, vol. 111, Leipzig, 1867. Nous utilisons la traduction française de Javal et Klein, Optique physiologique, Paris, Masson, 1867, p. 1000-1001.

37. Ibid., p. 579-580. 
VI. Helmholtz conçoit donc la fonction du signe/sensation dans une relation triadique entre le signe, le signifié et l'utilisateur/interprète du signe; en cela, son approche préfigure également le développement que connaîtra la sémiotique chez Peirce ${ }^{38}$. Toutefois, nous nous garderons bien de faire de Helmholtz un philosophe du langage; en bon kantien, celui-ci demeure un philosophe du règne des lois. Bien que l'on retrouve au cœur de son épistémologie une conception sémiotique des sensations, celles-ci sont pensées d'emblée sous la catégorie transcendantale de la causalité, de sorte que le reproche que lui adresse Cassirer semble tout à fait justifié :

Toute la théorie de la connaissance de Helmholtz est ancrée dans le concept de signe : le monde des phénomènes n'est pour lui rien de plus qu'un ensemble de signes qui n'ont aucune ressemblance avec leurs causes, les choses réelles, tout en leur étant légalement coordonnées de façon à pouvoir exprimer par eux-mêmes toutes les différences et tous les rapports des choses. Mais Helmholtz ne maintient pas avec une réelle rigueur systématique le primat ainsi reconnu au concept de symbole. Car au lieu d'intégrer et de subordonner désormais le problème de la causalité au problème général de la signification, il suit la voie inverse, et veut concevoir et expliquer la fonction même du signe comme une forme spéciale de relation causale ${ }^{39}$.

Helmholtz a pensé l'arbitraire du signe ${ }^{40}$, mais il ne l'a pas pensé pleinement : les sensations ne sont pas des

38. Ce fait est également noté par Ulrich Mayer, Semantischer... p. 11 et ss.

39. Philosophie der symbolischen Formen, vol. 111, Berlin, 1929. Réimpr., Darmstadt, Wissenschaftliche Buchgesellschaft, 1964. La Philosophie des formes symboliques, vol. 111, trad. C. Fronty, Paris, Minuit, 1972.

40. “La représentation que j'al d'une certaine table est juste et précise si je puis en déduire, avec exactitude et précision, les sensations que j'éprouverais en amenant mon coil ou ma main dans telle ou telle position déterminée par rapport à la table. Je ne puis concevoir aucun autre genre d'analogie entre une pareille idée et l'objet qu'elle représente. La première est le signe spirituel du 
signes dans l'acception contemporaine du terme, en raison de la relation causale postulée entre la sensation comme signe naturel et l'objet dont elle est à la fois le signe et l'effet. Sous réserve de prendre pleinement en compte l'arbitraire du signe, la conception sémiotique de Helmholtz offre des possibilités attrayantes pour un empirisme constructiviste ${ }^{41}$ qui serait justement d'allégeance non réaliste : elle permet de concevoir la composante factuelle de théories empiriques telles les théories physiques en tant que structures qui sont d'emblée des structures linguistiques. Le réel n'est que l'univers d'un discours où s'articulent des invariances et le langage, comme instance d'articulation signifiante, vient ainsi relayer la causalité dans son rôle transcendantal, si tant est que ce rôle soit inéluctable.

\section{Jean Leroux Département de philosophie Université d'Ottawa}

second.[...] La nature de ces signes mentaux importe peu, d'ailleurs, pourvu qu'ils forment un système qui présente une variété et un ordre suffisants; de même que les sons des mots d'une langue sont indifférents, pourvu qu'on en ait un nombre suffisant et qu'on possède assez de moyens de désigner leurs rapports grammaticaux.》 Handbuch..., p. 583.

41. Cassirer exprime ainsi le caractère constructif que prendra, à la suite de Helmholtz, la sémiotique de Hertz : “Fait remarquable, le même penseur dont les découvertes ont préparé et rendu possible, au plan du contenu, la nouvelle "image électrodynamique du monde", a aussi été l'auteur d'une "révolution du mode de pensée" à l'intérieur de la théorie physique. Heinrich Hertz est ce chercheur moderne qui, dans ses Principes de mécanique (1894) a accompli le plus tôt et le plus résolument la conversion de la "théorie de la connaissance comme copie en une pure théorie du symbole". Les concepts-clés de la science n'apparaissent plus, dès lors, comme des copies imitant un donné immédiat des choses, mais comme des projets constructifs de la pensée physique, projets dont la valeur et la portée théoriques ont pour unique condition un accord constamment renouvelé entre leurs conséquences nécessaires et ce que l'expérience permet d'observer. En ce sens, c'est tout le monde des concepts physiques qui peut maintenant, ainsi que fit Helmholtz dans sa théorie de la connaissance, se définir comme un monde de "purs signes".»Philosophie..., p. 33. 